\title{
Delay and inhibition of growth of Neisseria gonorrhoeae in vitro \\ Relationship of delayed incubation, penicillinase production, urine culture, and self-treatment
}

\author{
WILLIAM G WESTBROOK III \\ From the Microbiology Laboratory, USAF Hospital, Clark Air Base, Republic of the Philippines
}

SUMMARY Delay in the appearance of Neisseria gonorrhoeae on Thayer-Martin medium was associated with delay in incubation and with the use of urine samples from infected men for cultural diagnosis. Self-treatment among women was related to inhibition of growth of $N$ gonorrhoeae, particularly of non-penicillinase-producing gonococci ( $\beta$-lactamase). The difference in the time of appearance of penicillinase-producing and non-penicillinase-producing gonococci was significant in men but not in women.

\section{Introduction}

The laboratory plays a vital role in the diagnosis of most sexually transmitted diseases, but this role is particularly essential in gonorrhoea. The studies reported here were the result of a need to expand and refine laboratory techniques to diagnose gonorrhoea in a military and a local civilian population at Clark Air Base, Republic of Philippines. Gonorrhoea has been highly prevalent for years in this mobile and sexually promiscuous population, but the usual public health measures kept the infection under reasonable control until penicillinase-producing Neisseria gonorrhoeae (PPNG) strains emerged in the summer of $1976 .{ }^{12}$

Because PPNG strains could be identified only after cultural isolation, the bacteriological laboratory had to increase its workload several-fold to examine diagnostic and test-of-cure samples from the treatment clinic, gonorrhoea screening specimens from military and dependent women, and routine surveillance samples from local "hostesses" attending two local city public health clinics. Incubation of cultures from these clinics, located several miles away from the bacteriological laboratory, was delayed for one to four hours. It was necessary to determine the effect of delayed incubation of inoculated cultures on the recovery of

Address for reprints: Captain W G Westbrook, Clinical Laboratory, USAF Regional Hospital, MacDill Air Force Base, Florida 33608, USA

Received for publication 6 April 1979 both penicillinase-producing and non-penicillinaseproducing $N$ gonorrhoeae (non-PPNG).

An attempt was also made to increase surveillance for gonorrhoea in men, including those with no symptoms or signs of infection who could be contributing to the spread of gonorrhoea in this community. ${ }^{3}$ Because of real or imagined trauma caused by collection of urethral specimens with a wire loop or a calcium-alginate swab, men were reluctant to volunteer for urethral culture when they were asymptomatic. We decided to compare the sensitivity of unspun urine culture with the standard urethral culture method in men with and without symptoms of gonorrhoea with a view to substituting urine for urethral culture as has been reported. ${ }^{4}$

A final consideration was the effect of selftreatment on the recovery of $N$ gonorrhoeae by culture. Self-treatment was acknowledged to be common among women attending the city clinics and was thought to be contributing to the selection and spread of penicillin-resistant gonococci.

\section{Patients and methods}

Modified Thayer-Martin (MTM) medium was used for culture of $N$ gonorrhoeae throughout the study. ${ }^{5}$ Each batch of medium was prepared and tested locally for its ability to support the growth of gonococci.

The studies were carried out with the co-operation of: (a) men attending a military hospital clinic; (b) women attending a military hospital gynaecological clinic; (c) women attending a local city health clinic; and (d) local civilian men. 
COLLECTION OF SPECIMENS

Urethral specimens from men were obtained using calcium-alginate swabs. Endocervical culture specimens were collected with cotton swabs. Urethral specimens for culture were obtained from all men and endocervical specimens from all women. Urine specimens from selected men and women were obtained for culture and other tests. The aim was to secure the first $10 \mathrm{ml}$ of urine voided in the morning or after an interval of four hours since the last voiding. Urine was collected in a sterile plastic container and cultured within one minute. A sterile cotton swab was swirled in the urine to entrap bacteria and excess urine eliminated by rolling the swab against the wall of the container before inoculating the cultures. Swabbing in a " $Z$ " pattern across the culture medium was routine.

\section{CULTURAL TECHNIQUES}

All MTM plate inoculations took place within one minute of securing samples. Plates were placed in a $\mathrm{CO}_{2}$ candle jar within five minutes. Incubation at 36 $\pm 1^{\circ} \mathrm{C}$ took place within one hour in the case of men; within two hours in the case of women attending the gynaecological clinic; and within five hours in the case of women attending local health clinics.

\section{IDENTIFICATION}

All MTM plates were examined every 18-24 hours for a total of 96 hours. When typical gonococcal colonies were first seen, the incubation time was recorded. Typical colonies were presumptively identified as $N$ gonorrhoeae by oxidase reaction and examination of Gram-stained smears of suspected colonies. Owing to the large workload, presumed gonococci were not submitted to confirmatory tests. Penicillin sensitivity was determined by the standardised disc-diffusion susceptibility test $\left(10^{8}\right.$ colony-forming units per $\left.\mathrm{ml}\right)$ on enriched chocolate agar (less than $20 \mathrm{~mm}$ diameter zone of inhibition around a disc containing $10 \mu \mathrm{g}$ penicillin indicating resistance). ${ }^{6}$ Penicillinase production was detected by the chromogenic cephalosporin test. ${ }^{7}$ Positive and negative controls were used with all tests.

\section{BIOASSAY TECHNIQUE}

Mid-stream urine specimens from 512 consecutive women were examined for the presence of urinary bacterial inhibitory substances (UBIS). One drop of fresh urine from each of 12 patients was placed on the surface of a $150-\mathrm{mm}$ diameter bioassay plate. The bioassay plates contained Muller-Hinton agar seeded with Sarcina lutea (Micrococcus luteus) FDA strain 1001 , susceptible to $\leq 0.01 \mu \mathrm{g} / \mathrm{ml}$ of penicillin. All urine specimens tested were stored at $-10^{\circ} \mathrm{C}$ for possible retesting. Bioassay plates were capable of detecting UBIS in urine from two healthy men between one and eight hours after ingestion of $250 \mathrm{mg}$ of ampicillin by inhibiting growth of S lutea. UBIS was also detected in urine in these men 24 hours after taking $500 \mathrm{mg}$ of ampicillin by mouth.

Inhibition zones on bioassay plates were measured after 18-24 hours' incubation. When zones of inhibition coalesced or otherwise proved difficult to read, testing was repeated on plates allowing large areas for inhibition. Aliquots $(0.2 \mathrm{ml})$ of all urine specimens found to contain UBIS were mixed with an equal volume of commerically obtained penicillinase concentrate $\left(10 \times 10^{6}\right.$ kinetic units per ml) (Baltimore Biologicals, Cockeysville, Maryland) and incubated for 30 minutes at room temperature before being retested.

STATISTICAL ANALYSIS

Mean differences between the incubation times of the different groups cultured were tested by Student's $t$ test, assuming unequal variances. $\chi^{2}$ analysis was used to compare (a) the mean difference between the incubation times of the different groups; (b) the growth of $N$ gonorrhoeae at 24-hour intervals in the different populations studied; (c) the time of incubation needed to isolate $N$ gonorrhoeae from urine and urethral specimens when both were culture-positive in the same patient and whether or not symptoms of gonorrhoea were present; and (d) the effect of selftreatment on the recovery of $N$ gonorrhoeae.

\section{Results}

DELAYED INCUBATION

Of 63962 specimens examined, $5254(8 \cdot 2 \%)$ gave a positive result for $N$ gonorrhoeae according to the criteria. The mean time to appearance of colonies was directly related to the time-lapse between inoculation of MTM plates and incubation at $36 \pm 1^{\circ} \mathrm{C}$. Significantly longer incubation was needed to detect growth of gonococci isolated from women attending city clinics than from women attending the hospital gynaecological clinic and men attending the hospital outpatient clinic (Table I). A significantly longer time of incubation was needed for the isolation of PPNG in men than for non-PPNG; this was not true in either group of women.

PENICILLINASE-PRODUCING GONOCOCCI

Of the 1801 positive cultures from men, $304(21 \cdot 9 \%)$ grew PPNG strains. Of 3453 positive cultures from women, $683(19 \cdot 8 \%)$ grew PPNG strains. In men $96.9 \%$ of isolates were identified within 48 hours of incubation whereas only $81 \cdot 8 \%$ of the women attending the hospital gynaecological clinic had identifiable colonies at 48 hours (Table II). The figure for women attending the local health clinic was 
TABLE I Mean incubation time for growth and isolation of gonococci

\begin{tabular}{llllll}
\hline Clinic & $\begin{array}{l}\text { Type of } \\
\text { gonococcus }\end{array}$ & $\begin{array}{l}\text { Maximum delay } \\
\text { before incubation }\end{array}$ & $\begin{array}{l}\text { Mean incubation } \\
\text { time } \pm \text { SD) of } \\
\text { positive cultures }\end{array}$ & Significance* & Probability \\
\hline (1) City (F) & PPNG + non-PPNG & $5 \mathrm{hr}$ & $54 \cdot 9 \pm 15 \cdot 2 \mathrm{hrs}$ & (1) $v s(2) t=5 \cdot 5$ & $<0 \cdot 01$ \\
(2) Hospital (F) & PPNG + non-PPNG & $2 \mathrm{hr}$ & $45 \cdot 6 \pm 17 \cdot 5 \mathrm{hrs}$ & (2) $v s(3) t=7 \cdot 1$ & $<0 \cdot 01$ \\
(3) Hospital (M) & PPNG & $1 \mathrm{hr}$ & $32 \cdot 8 \pm 13 \cdot 5 \mathrm{hrs}$ & (3) $v s(4) t=2 \cdot 2$ & $<0 \cdot 05$ \\
(4) Hospital (M) & non-PPNG & $1 \mathrm{hr}$ & $31 \cdot 2 \pm 13 \cdot 0 \mathrm{hrs}$ & (2) $v s(4) t=8 \cdot 45$ & $<0 \cdot 01$ \\
\hline
\end{tabular}

*Student's $t$ test

TABLE II Time of incubation for cultures of $N$ gonorrhoeae

\begin{tabular}{lccccccc}
\hline \multirow{2}{*}{ Clinic } & \multicolumn{6}{c}{ No of PPNG/total positive cultures (cumulative \%) after } \\
\cline { 2 - 7 } & Total No & $24 \mathrm{hr}$ & $48 \mathrm{hr}$ & $72 \mathrm{hr}$ & $96 \mathrm{hr}$ & Total \\
\hline Hospital (M) & 8612 & $264 / 1301(72 \cdot 2)$ & $116 / 444(96 \cdot 9)$ & $13 / 47(99 \cdot 5)$ & $1 / 9(100)$ & $394 / 1801$ \\
Hospital (F) & 6863 & $8 / 33(30 \cdot 0)$ & $10 / 57(81 \cdot 8)$ & $1 / 18(98 \cdot 2)$ & $1 / 2(100)$ & $20 / 110$ \\
City (F) & 48487 & $19 / 107(3 \cdot 2)$ & $476 / 2385(74 \cdot 5)$ & $134 / 631(93 \cdot 4)$ & $34 / 220(100)$ & $663 / 3343$ \\
Total & 63962 & $291 / 1441(27 \cdot 4)$ & $602 / 2886(82 \cdot 3)$ & $148 / 801(97 \cdot 5)$ & $36 / 231(100)$ & $1077 / 5254$ \\
\hline
\end{tabular}

PPNG = penicillinase-producing $N$ gonorrhoeae

$74.5 \%$. The differences in time of incubation required to detect growth of $N$ gonorrhoeae in each of these groups was highly significant at 48 hours $\left(\chi_{2}^{2}=458.5, \mathrm{P}<0.001\right)$ and significant at 72 hours $\left(\chi_{2}^{2}=6 \cdot 2, \quad \mathrm{P}<0.05\right)$ but not before 48 hours $\left(\chi_{2}^{2}=1 \cdot 15\right)$ or after 72 hours $\left(\chi_{2}^{2}=2 \cdot 48\right)$.

URINE AND URETHRAL CULTURES (MEN)

All but 64 of the 985 paired urethral and urine samples from men were collected before the first urine of the day was passed or four hours or more after the last voiding.

Two hundred and eighty-four urethral specimens and 279 urine specimens gave positive results, that is a $\mathbf{9 8} \cdot \mathbf{2 \%}$ agreement. Urine sampling was associated with a significant delay in the appearance of gonococcal colonies $\left(\chi_{3}^{2}=38 \cdot 59, \mathrm{P}<0 \cdot 001\right)$ and of PPNG $\left(\chi_{1}^{2}=8 \cdot 09, \mathrm{P}<0 \cdot 02\right)$ compared with urethral

TABLE III Number of $N$ gonorrhoeae isolates identified at 24-hour intervals in the 279 cases for which both urine and urethral cultures gave positive results

\begin{tabular}{llc}
\hline \multirow{2}{*}{$\begin{array}{l}\text { Incubation time } \\
\text { (hrs) }\end{array}$} & \multicolumn{2}{c}{ No culture-positive (PPNG) } \\
\cline { 2 - 3 } & Urine & Urethra \\
\hline 24 & $163(34)$ & $229(48)$ \\
48 & $103(15)$ & $47(7)$ \\
72 & $11(4)$ & 3 \\
96 & $2(2)$ & 0 \\
Total & $279(55)$ & $279(55)$ \\
\hline
\end{tabular}

PPNG = penicillinase-producing $N$ gonorrhoeae sampling (Table III). This also applied to the 141 infected men whose symptoms were known irrespective of whether or not the organisms were PPNG strains (Table IV).

A total of 115 men with no urethral discharge or complaints of dysuria had both urine and urethral cultures performed. Seven $(6 \cdot 1 \%)$ of these men were infected with $N$ gonorrhoeae, which was detected by both urine and urethral culture.

Of a separate group of 111 local civilian men who had urine cultures performed later, five yielded nonPPNG isolates. Three of the five men denied having symptoms of gonorrhoea.

TABLE IV Number of $N$ gonorrhoeae isolates identified at 24-hour intervals in 141 cases for which both urine and urethral cultures gave positive results and symptoms were known

\begin{tabular}{lllc}
\hline & & \multicolumn{2}{c}{ No culture-positive (PPNG) } \\
\cline { 4 - 4 } & Incubation (hrs) & Urine & Urethra \\
\hline $\begin{array}{c}\text { Symptomatic } \\
(\mathrm{n}=134)\end{array}$ & 24 & $90(21)^{*}$ & $116(24)$ \\
& 48 & $40(5)$ & $17(4)$ \\
& 72 & $4(2)$ & 1 \\
$\begin{array}{c}\text { Asymptomatic } \\
(\mathrm{n}=7)\end{array}$ & 24 & 3 & \\
& 48 & $4(1)$ & 1 \\
& 72 & 0 & 0 \\
Total & & $141(29)$ & $141(29)$ \\
\hline
\end{tabular}

$\chi_{2}^{*}=11 \cdot 49, \mathrm{P}<0 \cdot 01$

PPNG = penicillinase-producing $N$ gonorrhoeae 
SELF-TREATMENT

Of 591 urine samples from female patients, 93 (15.7\%) contained UBIS (Table V). When the 93 samples were reacted with penicillinase concentrate, 73 failed to show UBIS suggesting that they had originally contained a $\beta$-lactam antibiotic-like penicillin or one of the cephalosporins. Two of these patients had been prescribed ampicillin by a physician within the previous 96 hours. The 20 patients with repeat positive results to the UBIS tests were considered to have taken antibiotics other than those mentioned.

Only two $(2 \cdot 2 \%)$ of the 93 patients with UBIS had positive endocervical culture results whereas 36 $(7 \cdot 2 \%)$ of 498 without evidence of self-treatment had positive results, a difference which was significant $\left(\chi_{2}^{2}=6 \cdot 133, P<0 \cdot 05\right)$. Of the two patients with UBIS who had positive culture results, both had PPNG strains. Of the 36 isolates from UBIS-negative patients, six $(16 \cdot 6 \%)$ were PPNG.

\section{Discussion}

The significant delay in recovering $N$ gonorrhoeae from women by culture beyond the usual 48 hours of incubation could be due to one or several of the following factors: (a) the delay in processing inoculated cultures before incubation at $36 \pm 1^{\circ} \mathrm{C}$; (b) the bacteriostatic activity of antimicrobial substances in the endocervical and vaginal secretions; (c) a smaller inoculum of gonococci from the endocervix compared with that from the urethra in men; and (d) a difference in the growth characteristics of gonococci isolated from women. A delay of two or more hours before incubation under $\mathrm{CO}_{2}$ at $36^{\circ} \mathrm{C}$ can produce a significant delay in the appearance of gonococcal colonies and a reduction in their size and number. ${ }^{8}$ This is the most logical explanation for the delay in recovering gonococci from women by culture in this study. Changes were made to ensure more prompt processing of cultures from female clinics. Even under the most ideal conditions, however, a small number of both PPNG and nonPPNG strains required more than 48 hours of incubation before growth could be detected.

TABLE V Comparison of urine specimens tested for growth inhibitory substances and isolation of $N$ gonorrhoeae by endocervical Thayer-Martin culture

\begin{tabular}{llll}
\hline & & \multicolumn{2}{l}{ No culture-positive } \\
\cline { 3 - 4 } Urine specimens & Culture & Non-PPNG (\%) & PPNG (\%) \\
\hline Non-inhibitory & 498 & $30(6 \cdot 0)$ & $6(1 \cdot 2)$ \\
Inhibitory* & 93 & $0+$ & $2(2 \cdot 2)$ \\
\hline
\end{tabular}

*Containing penicillin-like (73) or other (20) inhibitory substances $+\chi_{2}^{2}=6 \cdot 133, P<0 \cdot 05$
The culture of unspun urine gave results comparable to culture of urethral specimens obtained directly by swab in men with symptomatic and asymptomatic infections. The significantly longer incubation time needed to isolate $N$ gonorrhoeae from urine was probably due to the smaller number of gonococci contained in the urine. The collection of urine specimens for culture was more acceptable to patients than was that of urethral specimens; because of this the culture of the former might be more appropriate for screening large numbers of asymptomatically infected men. The need for prolonged incubation of urine cultures might be overcome by adding a small volume of concentrated MTM medium (minus agar) to the urine in the collection jar. Pre-incubation of this mixture might allow gonococci to multiply in the urine, thus increasing inoculum size.

The finding of penicillin-like substances in the urine of $12.4 \%$ of women attending the local city health clinic indicates the use of oral antibiotics for self-treatment or for prophylaxis of gonorrhoea in this population. Possibly the practice contributes to the selection and dissemination of drug-resistant strains of $N$ gonorrhoeae, including PPNG. These women had been told repeatedly that self-medication was dangerous and should not be practised and that there was no need for it, since all diagnostic and therapeutic services at the clinic were provided free. Thus continued patient education is necessary as well as improvements in the diagnosis and treatment of gonorrhoea in the Philippines.

I would like to thank Staff Sergeant Charles J Slabisak, CLA (ASCP), Mrs Adeluisa C Prater, RMT, Miss Elaine L Corre, RMT, Mr Doroteo B Dingcol, RMT, Mrs Cynthia C Henson, MT (ASCP), Mr Romeo V Reyes Jr, MT, and Miss Violeta A Villapando, MT, without whose help this study would not have been possible. My thanks to Colonel Robert R Smith, Lieutenant Colonel Donald G Wright, and Ignacia $R$ DeJesus, for their cooperation, to Dr R S Morton and Mr Akbar Zaidi for their assistance in preparing the manuscript, and, especially, to Peter L Perine, for his advice and close support.

Sarcina lutea, FDA strain 1001, was kindly supplied by J S Lewis, Center for Disease Control, Atlanta, Georgia, USA.

\section{References}

1. Ashford WA, Golash RG, Hemming VG. Penicillinaseproducing Neisseria gonorrhoeae. Lancet 1976; 2: 657-8.

2. Phillips I. $\beta$-lactamase-producing penicillin-resistant gonococcus. Lancet 1976; 2: 656-7. 
3. Handsfield, HH, Lipman TO, Harnisch JP, Tronca E, Holmes KK. Asymptomatic gonorrhea in men. N Engl J Med 1974; 290: $117-23$.

4. Feng WC, Medeiros AA, Murray ES. Diagnosis of gonorrhea in male patients by culture of uncentrifuged first-voided urine. JAMA 1977; 237: 896-7.

5. Thayer JD, Martin JE jun. An improved medium selective for cultivation of gonorrhoeae and meningitidis. Public Health Rep 1966; 81: 559-62.

6. Biddle JW, Swenson JM, Thornsberry S. Disc agar diffusion antimicrobial susceptibility tests with $\beta$-lactamase producing Neisseria gonorrhoeae. J Antibiot 1978; 31: 352-8.
7. O'Callaghan $\mathrm{CH}$, Morris A, Kirby SM, Shingler AH. Novel method for detection of $\beta$-lactamases by using a chromogenic cephalosporin substrate. Antimicrob Agents Chemother 1972; 1: 283-8.

8. Chapel TA, Smeltzer M, Dassel R. The effect of delaying incubation in a $\mathrm{CO}_{2}$-enriched environment on gonococci. Health Lab Sci Rep 1976; 13: 45-8. 